\title{
In memoriam Hans-Jürg Leisinger
}

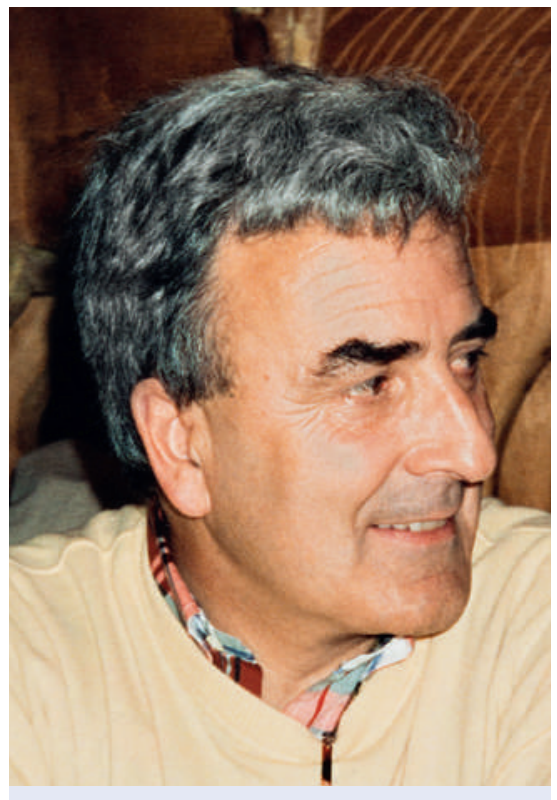

Prof. Dr Hans-Jürg Leisinger (1941-2012)
C'est avec une très grande tristesse que nous avons appris le décès du Professeur Hans-Jürg Leisinger qui s'est éteint le dimanche 7 octobre 2012 à l'âge de 71 ans.

Le Curriculum Vitae qu'il a adressé à la faculté de biologie et de médecine de l'Université de Lausanne pour postuler en tant que Prof ordinaire d'urologie en 1990 est un modèle de modestie. Nous gardons de lui le souvenir d'un homme de caractère généreux et bienveillant, disponible, respectueux vis-à-vis de ses proches et de tous les membres de son environnement professionnel, ouvert à toute innovation ou remise en question, encadrant et soutenant souvent avec enthousiasme les projets de ses collaborateurs. Grand travailleur, humaniste, Hans-Jürg Lesisinger assumait avec plaisir son rôle de patron du service d'urologie et de directeur du département des services de chirurgie et d'anesthésiologie.

Revenons sur son parcours, suivant les traces de son père, Fritz Leisinger, chirurgien chef de l'hôpital de Richterswil dans le canton de Zurich, Hans-Jürg se destine à la médecine et à la carrière chirurgicale. Son diplôme de médecin obtenu, il débute son cursus chez son père puis auprès du Prof. Ake Senning dans le service de chirurgie de l'hôpital universitaire de Zurich. Voici une petite anecdote au cours de cette période: D'esprit curieux et amateur de voyage, Hans-Jürg décide d'emmener à Berlin-Est, Marie-Claire, qui deviendra sa première épouse et la mère de ses deux filles Hélène et Caroline. Ces deux dangereux conspirateurs, nous sommes proches de mai 1968 , seront retenus 24 heures par la police est-allemande pour avoir introduit au-delà du mur, des journaux subversifs de l'ouest, lesquels ne servaient que d'emballages aux litres d'huile nécessaire à la marche capricieuse du moteur de sa «mini».

De retour en Suisse, il se tourne vers l'urologie et poursuit sa carrière chirurgicale auprès du Prof. Georges Mayor, éminent spécialiste de la chirurgie urologique en Suisse. Il obtient son titre FMH de chirurgie en 1974 et son titre FMH de spécialiste en Urologie en 1976. Il fera sa thèse de doctorat, sous la conduite du Prof. Ernst Zingg. Ce travail traite de la localisation atypique des adénomes parathyroïdiens dans les hyperparathyroidismes primaires. Il était entouré par des maîtres renommés et cherchera toujours à établir des contacts professionnels enrichissants.
Tout en poursuivant sa carrière de médecin-chef de l'urologie à l'hôpital de Schaffhouse, Hans-Jürg Leisinger développe dans le service de chirurgie expérimentale de l'Université de Zurich le concept de l'utilisation de l'intestin grêle dans le remplacement de la vessie en collaboration avec le Prof. Nils Koch de Göteborg. Comme en témoignent ses nombreuses publications, il sera dans le monde un des pionniers de la chirurgie du remplacement vésical par une poche intestinale continente. Ce travail d'habilitation lui permettra d'obtenir le titre de Professeur ordinaire et chef de service du service d'urologie au CHUV (Centre hospitalier Universitaire Vaudois), charge qu'il assume de 1992 à 2006.

Hans-Jürg Leisinger sera également nommé à la tête du département des services de chirurgie et d'anesthésiologie. Tous les collègues qui l'ont côtoyé soulignent son amabilité et sa pondération dans le traitement des affaires.

Il développe l'urologie du futur en associant l'activité clinique à une unité de recherche en urologie: l'URU. Cette dernière, toujours très active aujourd'hui, verra la naissance d'une start-up: Med-Discovery, entreprise dont il a toujours soutenu le développement avec enthousiasme. Nous tous lui sommes reconnaissants de son aide dans le développement de la chirurgie laparoscopique, de l'andrologie et de la cystoscopie de fluorescence sous l'égide d'une collaboration CHUV-UNIL-EPFL.

Au plan national et international. Hans-Jürg Leisinger sera président et secrétaire de la Société suisse d'urologie de nombreuses années. Il deviendra membre d'honneur de nombreuses sociétés. Ses fonctions dans les instances exécutives de l'association européenne d'urologie l'amèneront à tenir la présidence du Congrès annuel de l'EAU à Genève en 2001.

Soucieux de l'aide au développement, Hans-Jürg Leisinger s'engage dans la création d'unité d'urologie au Togo et à Cantho au Vietnam.

Au plan personnel, féru de ski alpin dans sa jeunesse, Hans-Jürg Leisinger se révèle non seulement un amateur des bonnes tables, mais un fin cuisinier, un mélomane averti et il développe une passion pour l'histoire de l'art avec une préférence marquée pour l'art moderne. Il partagera cette passion avec sa seconde épouse Luzi, sa première épouse étant décédée tragiquement peu de temps après son arrivée au CHUV.

Nous perdons non seulement un maître, mais un compagnon et un ami. Merci pour tout, Hans-Jürg.

Prof. Dr Patrice Jichlinski,

Médecin-chef du service d'Urologie, CHUV-Lausanne 